\title{
Fast bi-exponential fluorescence lifetime imaging analysis methods
}

\author{
David Day-Uei Li, ${ }^{1, *}$ Hongqi Yu, ${ }^{1,2}$ and Yu Chen ${ }^{3}$ \\ ${ }^{1}$ Centre for Biophotonics, SIBPS, University of Strathclyde, Glasgow, G4 ORE, Scotland, UK \\ ${ }^{2}$ School of Electronic Science and Engineering, National University of Defense Technology, Changsha 410073, China \\ ${ }^{3}$ Department of Physics, University of Strathclyde, Glasgow G4 ONG, Scotland, UK \\ *Corresponding author: David.Li@strath.ac.uk
}

Received November 12, 2014; revised November 26, 2014; accepted December 1, 2014;

posted December 2, 2014 (Doc. ID 226735); published January 22, 2015

\begin{abstract}
A new hardware-friendly bi-exponential fluorescence lifetime imaging (FLIM) algorithm has been proposed. Compared to conventional FLIM software, the proposed algorithms are noniterative offering direct calculation of lifetimes and therefore suitable for real-time applications. They are applicable to single-channel or 2D multichannel time-correlated single-photon counting (TCSPC) systems. The proposed methods have been tested on both synthesized and realistic FLIM data, and we have compared their performances with other recently proposed nonfitting bi-exponential techniques showing promising applications in future massive solid-state TCSPC imagers. (c) 2015 Optical Society of America

OCIS codes: (030.5260) Photon counting; (040.1345) Avalanche photodiodes (APDs); (110.0180) Microscopy; (170.3650) Lifetime-based sensing; (170.6920) Time-resolved imaging.

http://dx.doi.org/10.1364/OL.40.000336
\end{abstract}

A fluorescence lifetime imaging microscopy (FLIM) system does not only acquire the intensity but also the temporal decay of the fluorescence [1]. As lifetime characteristics of fluorophores are usually sensitive to their environment, FLIM can be a powerful tool to monitor physiological parameters such as $\mathrm{pH}, \mathrm{O}_{2}, \mathrm{Ca}^{2+}$, or image cellular protein-protein interactions that link to diseases or tumors [2-4].

There are frequency-domain and time-domain FLIM systems. In this Letter we will only discuss nonfitting time-domain methods applicable to highly parallel single-photon avalanche diode (SPAD) arrays [ [ $\underline{-}-7]$. Time-domain FLIM usually employs time-gated cameras [ $\underline{6}, \underline{8}-10]$ or time-correlated single-photon counting (TCSPC) modules $[\underline{1}, \underline{3}]$. Only the former technique can provide high-speed acquisition. Grant $e t$ al. used the two-gate rapid lifetime determination (RLD) method to obtain fast FLIM imaging ( $\sim 10 \mathrm{fps})$, but the frame rate drops significantly when multiple gates (No. of gates $=M$ ) are applied to image samples exhibiting significant variations in lifetimes across the field of view [8,9]. Moreover, multigate methods require iterative nonlinear fitting algorithms for lifetime analysis. Although gating techniques provide faster imaging than traditional TCSPCs, at the same acquisition they are unable to provide the raw imaging data that most biologists feel comfortable with. To record a complex decay profile, for example one with a gated imager, a large number of intensity images at different delays $(M \gg 1)$ from the excitation pulse is acquired. A narrow sliding gate (gate width $\sim$ hundreds of picoseconds) is usually used to ensure enough timing resolution, but the photon efficiency is extremely low. For biologists a TCSPC with FLIM software is still the gold standard. Most TCSPC-based FLIM experiments are conducted by choosing a pixel dwell time to produce a frame rate around $0.2-0.5$ frames-per-minute to avoid photobleaching. Such an acquisition might be enough for observing drug efficacy in cancer, but surely impossible for imaging rapid cellular dynamics within seconds.
Recent advances in SPADs have shown a great potential to provide a step-change in FLIM techniques [5-7]. SPADs can be fabricated in 2D arrays and integrated with digital circuits in a single chipset using a power supply compatible with commercial field programmable gate array (FPGA) chips without using any cooling modules and high-voltage power supplies (usually required in CCD systems). The data throughput of the latest SPAD imagers can be easily higher than $50 \mathrm{~Gb} / \mathrm{s}$, and it is impossible to process the raw data without introducing any data compression similar to the fast FLIM processors [7]. These hardware friendly algorithms mainly serve to (1) compress the raw data to enhance the frame rate and to release the need for high-speed serial links between the camera and a workstation and (2) generate fast FLIM imaging with the minimum latency between the data collection and image generation for real-time applications. In this Letter we will propose new hardware friendly algorithms and compare their performances with some recently published nonfitting algorithms [11-13].

Assume that the fluorescence is a bi-exponential decay, and the measured density is $f(t)=K \cdot\left[f_{D}\right.$. $\left.\exp \left(-t / \tau_{F}\right)+\left(1-f_{D}\right) \cdot \exp \left(-t / \tau_{D}\right)\right] \cdot u(t) \cdot \tau_{F}, \tau_{D}$ are the lifetimes, $K$ is the pre-scalar, $f_{D}$ the proportion, $u(t)$ the step function, and we neglect the instrumental response function (IRF) and background noise as the model described in [12]. The assumption allows a proper comparison with other algorithms and provides intuitive insights for choosing a proper strategy in system design and data acquisition. The background noise can be corrected in most experiments without affecting the precision as long as they are smaller than the true signals.

Figure 1(a) shows the fluorescence density $f(t)$. Usually in an FLIM experiment, the measurement window $T$ is divided into $M(M>2)$ channels with the channel width of $h . N_{j}$ is the photon count in the $j$ th channel, and the photon count in the first channel is 

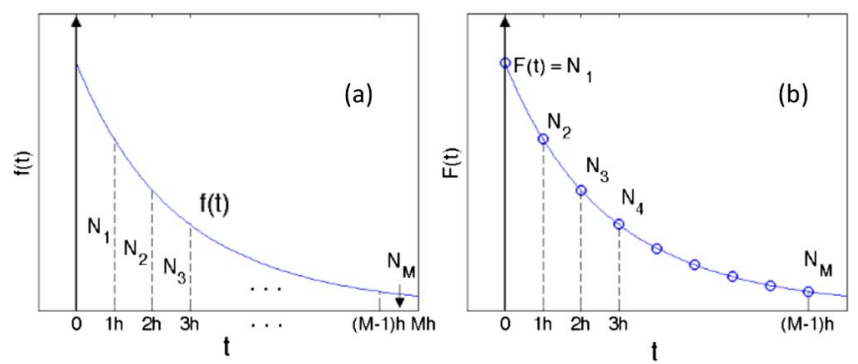

Fig. 1. (a) Decay function $f(t)$ and (b) sampled $F(t)$.

$$
N_{1}=\int_{0}^{h} f(t) \mathrm{d} t \cong K f_{D} h+K\left(1-f_{D}\right) h=K \cdot h,
$$

when $h \ll 2 \tau_{F}\left(\tau_{F}<\tau_{D}\right)$. This condition sustains in most commercially available TCSPC systems. If the condition cannot be met, calibration procedures can be applied to fix the bias. From Eq. (1), we can obtain

$$
\begin{aligned}
N_{j} & =\int_{(j-1) h}^{j h} f(t) \mathrm{d} t \\
& \cong K h f_{D} e^{-(j-1) h / \tau_{F}}+K h\left(1-f_{D}\right) e^{-(j-1) h / \tau_{D}} \\
& =N_{1} f_{D} e^{-(j-1) h / \tau_{F}}+N_{1}\left(1-f_{D}\right) e^{-(j-1) h / \tau_{D}},
\end{aligned}
$$

$j=2, \ldots, M$. The goal is equivalent to resolving a signal $F(t)=N_{1} f_{D} \exp \left(-t / \tau_{F}\right)+N_{1}\left(1-f_{D}\right) \exp \left(-t / \tau_{D}\right), \quad$ with the signal sampled at $t_{j}=(j-1) h$ as shown in Fig. $1(\mathrm{~b})$. Assuming $T \gg \tau_{D}$, we can obtain

$$
\begin{aligned}
\int_{0}^{(M-1) h} F(t) \mathrm{d} t & \cong N_{1} f_{D} \tau_{F}+N_{1}\left(1-f_{D}\right) \tau_{D} \\
& \cong h \cdot \sum_{j=1}^{M}\left(C_{j} \cdot N_{j}\right)=h \cdot N \\
N & \equiv \sum_{j=1}^{M}\left(C_{j} \cdot N_{j}\right),
\end{aligned}
$$

where $\mathbf{C}_{j}, j=1,2, \ldots, M$, are coefficients for Simpson's or Romberg's integration rule. Similarly, we can obtain Eq. (4),

$$
\begin{aligned}
\int_{0}^{(M-1) h} t \cdot F(t) \mathrm{d} t & \cong N_{1} f_{D} \tau_{F}^{2}+N_{1}\left(1-f_{D}\right) \tau_{D}^{2}, \\
& \cong h \cdot \sum_{j=1}^{M}\left(C_{j} \cdot t_{j} \cdot N_{j}\right)=h \cdot X, \\
X & \equiv\left(C_{j} \cdot t_{j} \cdot N_{j}\right) .
\end{aligned}
$$

Similar to the phasor approach (Phasor) and moment method (MoM) $[\underline{12}, \underline{13}]$, we can fix $\tau_{D}$ to obtain $\tau_{F}$ and $f_{D}$ as Eqs. (ㅁ) and (ㅁ),

$$
\begin{gathered}
\tau_{F}=\frac{\tau_{D} N-X}{\tau_{D} K-N}, \\
f_{D}=\frac{\tau_{D} K-N}{K\left(\tau_{D}-\tau_{F}\right)} .
\end{gathered}
$$

Compared with the single-exponential center-of-mass method (CMM) [7], Eqs. (5) and (6) are able to solve bi-exponential decays by just including an extra known $N_{1}$. We call this new method bi-decay CMM (BCMM), denoted as $\mathrm{BCMM}_{1}$. Unlike the previously published minimal fraction of interacting donor method $\left(m f_{D}\right)$ giving only underestimated $f_{D}[11], \mathrm{BCMM}_{1}$ provides both accurate $\tau_{F}$ and $f_{D}$. It is much simpler than Phasor and MoM and more hardware friendly, for example it only requires counters and accumulators in FPGAs like CMM [7]. We define

$$
\begin{aligned}
N^{\prime} & \equiv \int_{0}^{T} f(t) \mathrm{d} t, \quad X^{\prime} \equiv \int_{0}^{T} t \cdot f(t) \mathrm{d} t, \quad Y^{\prime} \equiv \int_{0}^{T} t^{2} \cdot f(t) \mathrm{d} t \\
u & =\int_{0}^{T} \cos (\omega t) \cdot f(t) \mathrm{d} t / N^{\prime}, \quad v=\int_{0}^{T} \sin (\omega t) \cdot f(t) \mathrm{d} t / N^{\prime}
\end{aligned}
$$

where $\omega$ is the laser repetition angular frequency. All methods are re-derived and summarized in Table 1 . When $\tau_{D}$ is unknown, Eq. (7) can be included to provide an extra condition for solving $\tau_{D}$

$$
\begin{aligned}
\int_{0}^{(M-1) h} \frac{t^{2}}{2} \cdot F(t) \mathrm{d} t & \cong N_{1} f_{D} \tau_{F}^{3}+N_{1}\left(1-f_{D}\right) \tau_{D}^{3} \\
& \cong h \cdot \sum_{j=1}^{M}\left(C_{j} \cdot \frac{t_{j}^{2}}{2} \cdot N_{j}\right) \\
& =h \cdot Y, Y \equiv \sum_{j=1}^{M}\left(C_{j} \cdot \frac{t_{j}^{2}}{2} \cdot N_{j}\right) .
\end{aligned}
$$

As $N_{1}$ is included there is no need to use the third moment (requiring a much bigger $T / \tau_{D}$ ratio) as the early MoM work [14] greatly reduces the complexity (denoted as $\mathrm{BCMM}_{2}$ hereafter). Similar to the MoM [12], bias correction is required for $\mathrm{BCMM}_{2}$ if $T / \tau_{D}$ is not large enough. Ignoring lengthy derivations here, Table $\underline{1}$ also lists the $\mathrm{BCMM}_{2}$ with both $\tau_{F}$ and $\tau_{D}$ unknown. To compare BCMM with Phasor and MoM, Monte Carlo simulations were carried out with $\tau_{D}=2.5 \mathrm{~ns}, \quad M=81$, $T=18 \mathrm{~ns}, 0.1<f_{D}<1.0$ and $0.2 \mathrm{~ns}<\tau_{F}<1.8 \mathrm{~ns}$. The integrals $N^{\prime}, X^{\prime}, Y^{\prime}, u$, and $v$ can be obtained from

$S \cong h \cdot \sum_{j=1}^{M}\left[g\left(t_{j}+\frac{h}{2}\right) \cdot N_{j}\right] \begin{gathered}g(t)=1, t, t^{2}, \cos (\omega t), \sin (\omega t), \\ \text { for } S=N^{\prime}, X^{\prime}, Y^{\prime}, u N^{\prime}, v N^{\prime}\end{gathered}$

Figures 2(a), 2(c), and 2(e) show the precision $(F$ value) plots. ( $F$-value is defined as $F \equiv N_{C}^{0.5} \sigma g / g$ [7], $g=\tau_{F}$ or $f_{D}$, and $\sigma g$ is the standard deviation of g.) Figures 2(b), 2(d), and 2(f) show the bias $\left(\left(=\Delta g / g, g \equiv\left|\langle g\rangle-g_{\text {real }}\right|\right)\right)$ for different methods. BCMM favors a smaller $\tau_{F}$, whereas Phasor and MoM favor a larger $\tau_{F}$. Figures $2(\mathrm{c})$ and $2(\mathrm{~d})$ show the precision and bias plots (for $\overline{\tau_{F} \text { ) for } \mathrm{BCMM}_{2}}$ using the Romberg's rule. $\mathrm{BCMM}_{2}$ is slightly less efficient than $\mathrm{BCMM}_{1}$, but it does not require any fixed parameter. MoM is the most biased among all methods, regardless of $M$, and worse than $\mathrm{BCMM}_{2}$ although they both use the second moment. The integral of the second moment, $Y^{\prime}$, in $[T, \infty]$ used in 
Table 1. $\tau_{F}$ and $f_{D}$ for $m f_{D}$, Phasor, MoM, and the Proposed BCMM

\begin{tabular}{|c|c|c|}
\hline Methods & $\tau_{F}$ & $f_{D}$ \\
\hline$m f_{D}[11]$ & N/A & Minimum $f_{D}=\left(\tau_{D}^{2} N^{\prime 2}-\tau_{D} X^{\prime} N^{\prime}\right) /\left(0.5 X^{\prime}-\tau_{D} N^{\prime}\right)^{2}$ \\
\hline Phasor [12] & $\frac{1-u-v \tau_{D} \omega}{\omega\left(v-u \tau_{D} \omega\right)}$ & $\frac{\tau_{D}\left(1+\tau_{F}^{2} \omega^{2}\right)\left(1-u-u \tau_{D}^{2} \omega^{2}\right)}{\left.\tau_{D}-\tau_{F}\right)\left(1-u-u \tau_{F}^{2} \omega^{2}-\tau_{F} \tau_{D} \omega^{2}-u \tau_{D}^{2} \omega^{2}-u \tau_{\tau}^{2} \tau_{D}^{2} \omega^{4}\right)}$ \\
\hline MoM $[\underline{12,} \underline{13}]$ & $\left(\tau_{D} X^{\prime}-0.5 \cdot Y^{\prime}\right) /\left(\tau_{D} N^{\prime}-X^{\prime}\right)$ & $\tau_{D}\left(\tau_{D} N^{\prime}-X^{\prime}\right) /\left\{\left(\tau_{D}-\tau_{F}\right)\left[\left(\tau_{D}+\tau_{F}\right) N^{\prime}-X^{\prime}\right]\right\}$ \\
\hline $\mathrm{BCMM}_{1}$, Eqs. (ㅁ) and ( $\left.\underline{6}\right)$ & $\left(\tau_{D} K-N\right) /\left[K\left(\tau_{D}-\tau_{F}\right)\right]$ & $\left(\tau_{D} K-N\right) /\left[K\left(\tau_{D}-\tau_{F}\right)\right]$ \\
\hline \multirow[t]{2}{*}{$\mathrm{BCMM}_{2} \tau_{D}$ Unknown } & $\tau_{F}=0.5 \cdot\left[G-\sqrt{G^{2}-4(N \cdot G-X) / K}\right]$ & $f_{D}=\left(K \cdot \tau_{D}-N\right) /\left[K \cdot\left(\tau_{D}-\tau_{F}\right)\right]$ \\
\hline & $\tau_{D}=0.5 \cdot\left[G+\sqrt{G^{2}-4(N \cdot G-X) / K}\right.$ & where $G=(K \cdot Y-N \cdot X) /\left(K \cdot X-N^{2}\right)$ \\
\hline
\end{tabular}

MoM cannot be ignored and needs to be corrected [12]. Figures 3(a) and 3(c) show the precision plots for $\bar{f}_{D}$, $F\left(f_{D}\right)$, whereas Figs. $3(\mathrm{~b})$ and $3(\mathrm{~d})$ show the bias plots,

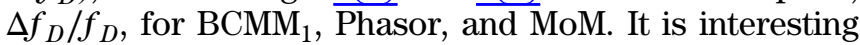
that the precision of $f_{D}$ seems better than that of $\tau_{F}$. Similar to the observations above, the BCMM favors a smaller $\tau_{F}$ and the Phasor and MoM are optimized around $\tau_{F} \sim 1 \mathrm{~ns}$ when $\tau_{D}$ is $2.5 \mathrm{~ns}$. Again, the MoM results are the most biased among all approaches. Figures 2(a), $2(\mathrm{~b}), \underline{3(\mathrm{a})}$, and $3(\mathrm{~b})$ also show the $\mathrm{BCMM}_{1}$ results using the Romberg's rule, which are comparable to the results obtained by the Simpson's rule. The Simpson's rule is useful when a gated or TCSPC system only has a limited number of temporal channels. For implementations in embedded hardware similar to [15], its complexity is similar to the Romberg's rule. To test BCMM two-photon
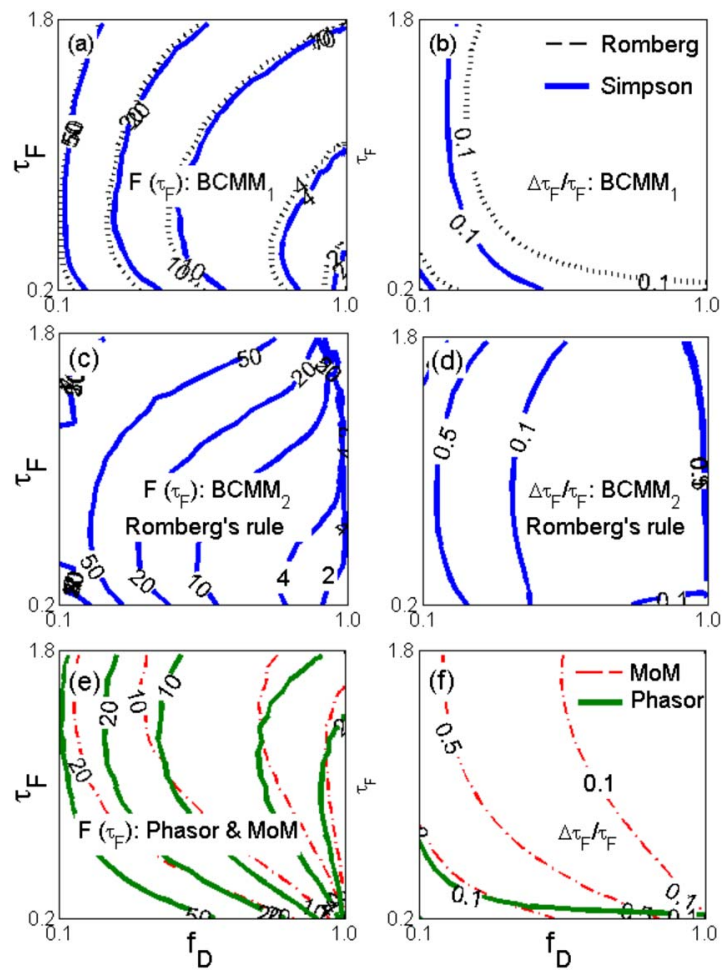

Fig. 2. (a), (c), and (e) are precision plots for $\tau_{F}$, whereas (b), (d), and (f) are accuracy plots for different methods.
FLIM was performed on Cy5-ssDNA-GNRs (gold nanorods) labeled Hek293 cells. The synthesis of GNR-based RNA nanoprobes was described elsewhere [4]. Briefly, GNRs were functionalized with thiolated oligonucleotides (ssDNA) labeled with Cy5 through ligand exchange and salting aging process [16]. Following incubation with Cy5-ssDNA-GNRs, Hek293 cells were washed and fixed with paraformaldehyde. FLIM was performed using a confocal microscope (LSM 510, Carl Zeiss) with a TCSPC module (SPC-830, Becker \& Hickl GmbH). A femtosecond Ti:sapphire laser (Chameleon, Coherent) was tuned at $800 \mathrm{~nm}$. The laser pulse has a repetition rate of $80 \mathrm{MHz}$ and duration less than $200 \mathrm{fs}$. The fluorescence of Cy5 was largely quenched by GNRs because of energy transfer arising from the hairpin structure of ssDNA [4]. Hybridization of nanoprobes with target RNA in cells opens the hairpin structure and results in significant increase in fluorescence intensity. The effects of the IRF can be neglected as a proper measurement window after the peak of the histogram was selected without applying deconvolution. Figure 4(a) depicts the intensity image of Hek293 cells. Figures $4(\mathrm{~b})-4(\mathrm{~d})$ show $f_{D}$ images obtained by the Phasor, MoM, and $\mathrm{BCMM}_{1}$, respectively. Figures $\underline{4(\mathrm{e})}$ and $\underline{4(\mathrm{f})}$ show $\tau_{D}$ and $f_{D}$ images obtained by
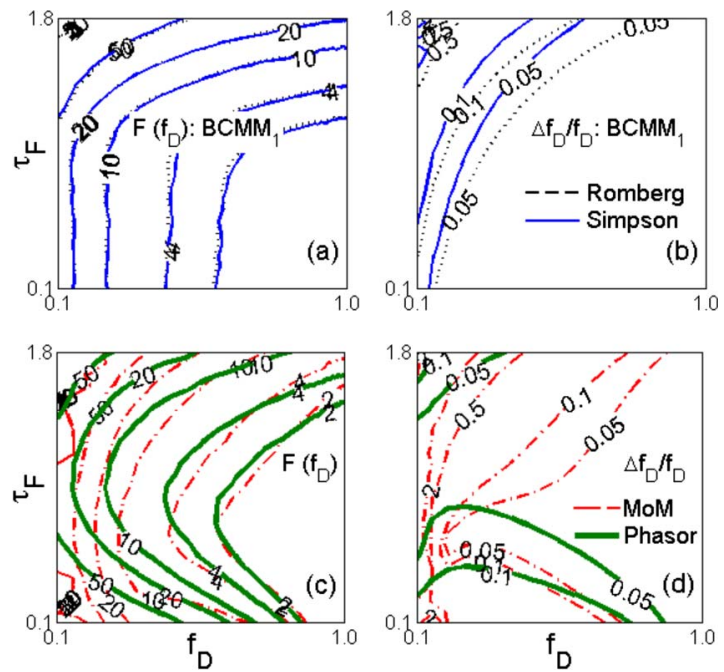

Fig. 3. (a) and (c) are precision plots, whereas (b) and (d) are accuracy plots for $\mathrm{BCMM}_{1}$, Phasor, and MoM. 

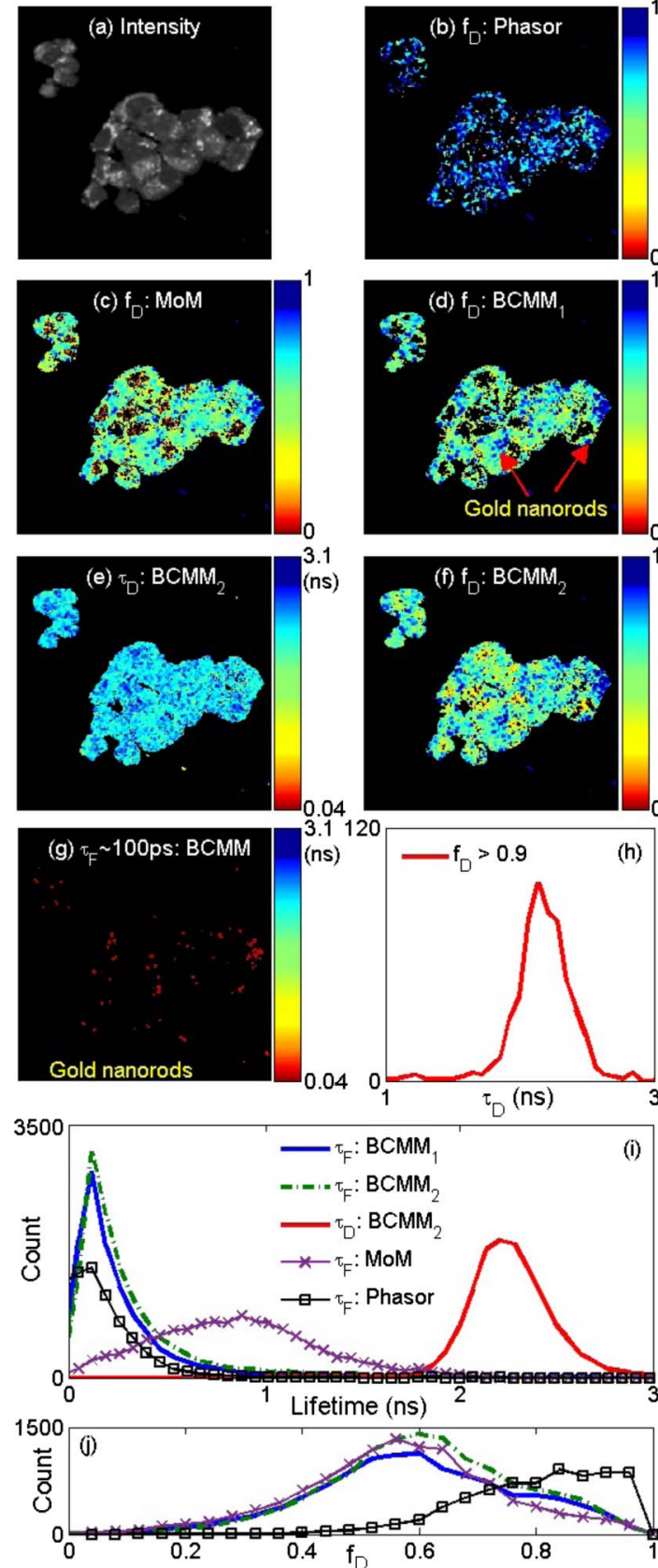

Fig. 4. (a) Intensity and (b)-(g) lifetime images using different methods; (h), (i), and (j) are lifetime and $f_{D}$ histograms.

$\mathrm{BCMM}_{2}$ with a simple look-up table correcting the bias. A measurement window $T \sim 5 \tau_{D}$ was used for acquisition. The average lifetime of the background free of GNRs, $\tau_{D}=2.3 \mathrm{~ns}$, was set for Phasor, $\mathrm{BCMM}_{1}$, and MoM. Phasor obtains a slightly bigger $f_{D}$, Fig. 4(b). Both $\mathrm{BCMM}_{1}$ and $\mathrm{BCMM}_{2}$ obtained $\tau_{F} \sim 100$ ps as in Fig. $\underline{4(\mathrm{~g})}$, consistent with the typical lifetime of two-photon luminescence of GNRs as reported previously [4]. Figure $4(\mathrm{~h})$ shows lifetime histograms for $\tau_{D}$ with GNR $s$ $\left(\tau_{D} \sim 2.16 \pm 0.20 \mathrm{~ns}\right)$. The lifetime reduction of Cy5 is due to the energy transfer to GNRs [4]. Figures 4(i) and $4(\mathrm{j})$ show lifetime and $f_{D}$ histograms or different algorithms. The MoM shows a significant bias $\left(\tau_{F, \text { MoM }} \sim 0.8 \mathrm{~ns}\right)$ if the bias is not corrected, consistent with the previous simulations. The bias can be corrected using the method introduced in [12] or a look-up table for faster corrections [7]. Figures $\underline{4(\mathrm{~b})}, 4(\mathrm{i})$, and $4(\mathrm{j})$ show that Phasor is biased when $\tau_{F} \ll \tau_{D}$ making it difficult to locate GNRs precisely.

In summary, we have proposed a hardware friendly biexponential algorithm, BCMM, suitable for high-speed FLIM analysis. It is much similar and faster, and its performance was demonstrated and compared with other previously reported methods. The results show that the BCMM can be a powerful tool suitable for real-time applications. Similar to the previously proposed CMM processors [7], BCMM can be embedded in hardware with minimal extra resources.

The authors would like to acknowledge the Royal Society (RG110438), BBSRC (BB/K013416/1), G. Wei, J. Sutter, W. Li, and R. Y. M. M. Qotob for this work.

\section{References}

1. W. Becker, J. Microsc. 247, 119 (2012).

2. Y. Sun, N. Hatami, M. Yee, J. Phipps, D. S. Elson, F. Gorin, R. J. Schrot, and L. Marcu, J. Biomed. Opt. 15, 056022 (2010).

3. M. Nobis, E. McGhee, J. Morton, J. Schwarz, S. Karim, J. Quinn, M. Edward, A. Campbell, L. McGarry, T. Jeffry Evans, V. Brunton, M. Frame, N. Garragher, Y. Wang, O. Sansom, P. Timpson, and K. Anderson, Cancer Res. 73, 4674 (2013).

4. Y. Zhang, G. Wei, J. Yu, D. Birch, and Y. Chen, Faraday Discuss., doi: 10.1039/C4FD00199K (2014).

5. R. M. Field, S. Realov, and K. L. Shepard, IEEE J. Solid-State Circuits 49, 867 (2014).

6. S. Burri, Y. Maruyama, X. Michalet, F. Regazzoni, C. Bruschini, and E. Charbon, Opt. Express 22, 17573 (2014).

7. D. D.-U. Li, J. Arlt, D. Tyndall, R. Walker, J. Richardson, D. Stoppa, E. Charbon, and R. Henderson, J. Biomed. Opt. 16, 096012 (2011).

8. D. Grant, J. McGinty, E. McGhee, T. Bunney, D. Owen, C. B. Talbot, W. Zhang, S. Kumar, I. Munro, P. Lanigan, G. Kennedy, C. Dunsby, A. Magee, P. Courtney, M. Katan, M. Neil, and P. French, Opt. Express 15, 15656 (2007).

9. T. Omer, L. Zhao, X. Intes, and J. Hahn, J. Biomed. Opt. 19, 086023 (2014).

10. M. Elangovan, R. N. Day, and A. Periasamy, J. Microsc. 205, 3 (2002).

11. S. Padilla-Parra, N. Auduge, M. Coppey-Moisan, and M. Tramier, Biophys. Rev. 3, 63 (2011).

12. A. Leray, S. Padilla-Parra, J. Roul, L. Héliot, and M. Tramier, PLoS ONE 8, e69335 (2013).

13. I. Gregor, B. Krämer, F. Koberling, R. Erdmann, J. Enderlein, M. Wahl, and S. Fore, Proc. SPIE 7903, 790330 (2011).

14. I. Isenberg and R. D. Dyson, Biophys. J. 9, 1337 (1969).

15. D.-U. Li, J. Arlt, J. Richardson, R. Walker, A. Buts, D. Stoppa, E. Charbon, and R. Henderson, Opt. Express 18, 10257 (2010).

16. A. Wijaya, S. B. Schaffer, I. G. Pallares, and K. HamadSchifferli, ACS Nano 3, 80 (2009). 